\title{
Elimination of loading reverberation in the split Hopkinson torsional bar
}

\author{
Q. Xue, L. T. Shen, and Y. L. Bai \\ Laboratory For Non-linear Mechanics of Continuous Media, Institute of Mechanics, \\ Chinese Academy of Sciences, Beijing 100080, People's Republic of China
}

(Received 1 June 1995; accepted for publication 24 July 1995)

\begin{abstract}
The loading reverberation is a multiple wave effect on the specimen in the split Hopkinson torsional bar (SHTB). Its existence intensively destroys the microstructure pattern in the tested material, and therefore, interferes with the study correlating the deformed microstructure to the macroscopic stress--strain response. This paper discusses the problem of the loading reverberation and its effects on the post-mortem observations in the SHTB experiment. The cause of the loading reverberation is illustrated by a stress wave analysis. The modification of the standard SHTB is introduced, which involves attaching two unloading bars at the two ends of the original main bar system and adopting a new loading head and a couple of specially designed clutches. The clutches are placed between the main bar system and the unloading bars in order to lead the secondary loading wave out of the main bar system and to cut off the connection in a timely manner. The loading head of the standard torsional bar was redesigned by using a tube-type loading device associated with a ratchet system to ensure the exclusion of the reflected wave. Thus, the secondary loading waves were wholly trapped in the two unloading bars. The wave recording results and the contrasting experiments for examining the post-mortem microstructure during shear banding both before and after the modification highly support the effectiveness of the modified version. The modified SHTB realizes a single wave pulse loading process and will become a useful tool for investigating the relation between the deformed microstructure and the macroscopic stress-strain response. It will play an important role especially in the study of the evolution of the microstructure during the shear banding process. 1995 American Institute of Physics.
\end{abstract}

\section{INTRODUCTION}

The split Hopkinson torsional bar (SHTB) is one of the most widely used instruments for determining the mechanical behavior of materials under high strain rate loading. Baker and Yew (1966) ${ }^{1}$ first introduced this apparatus, based on Kolsky's principle. ${ }^{2}$ Since then, a number of investigators, such as Campbell and Dowling, ${ }^{3}$ Nicholas, ${ }^{4}$ Duffy et al.,${ }^{5}$ Frantz and Duffy, ${ }^{6}$ Lipkin et al. ${ }^{7}$ Gilat and Pao, ${ }^{8}$ etc., have improved and expanded this technique. Their work mainly focused on improving the loading type and configuration of the stress wave and developing a new version for the study of the strain rate history effect (incremental or decremental strain rate tests). All these modifications made the SHTB a powerful tool for investigating the dynamic behavior and shear localization phenomenon of various materials.

Thermoplastic shear localization has been studied extensively as an important mechanism and as a precursor to fracture failure of ductile materials for many years. The further exploration of the phenomenon is aimed at understanding the inherent mechanism controlling the shear banding. "A critical need is for an improved understanding of the relation of localization to the microstructure of the material." Special attention has been focused on how the microstructures and microdamages of the materials evolve throughout the shear banding process, and how these evolving microstructures determine the macroscopic stress-strain response of the material. A more precise and quantitative measurement of the evolving microstructure in shear localization has become a prerequisite for all research. However, the standard SHTB cannot provide the required quantitative post-mortem observation of the deformed microstructure due to its loading wave reverberation. The stress wave reverberation resulting from the reflection of the loading wave in the bar system imposes multiple loadings on the specimen and destroys the real deformed microstructure after the first loading pulse. Thus, it seriously impairs the ability to correlate the evolving microstructure to the transient shear stress-strain response. Due to this problem, Xue, Bai, and Shen $(1992)^{10}$ partially modified the standard SHTB by using an unloading bar and a specially designed clutch. The reflection of the transmitted wave in the SHTB is then led out of the bar system after the first loading. Accurate measurement of the microstructure of shear banding can be ensured by controlling the amplitude of the loading wave, so that the reflected waves cannot cause secondary plastic deformation in the specimen. Obviously, the control of the loading wave limits the loading strain rate. Therefore, the complete elimination of loading reverberation requires improvements throughout the standard SHTB.

In the present paper, a complete modification of the SHTB to obtain a single pulse loading is introduced. By attaching unloading devices, the newly designed loading system can lead both the reflected wave and the transmitted wave out of the main bar system and ensure the accurate observation of the deformed microstructure that is subjected only to a single stress pulse. This modification makes it possible to record the correlation of the evolution of the microstructure to the transient shear stress strain. The observations of shear localization in a low carbon steel have confirmed the success of this modified SHTB.

\section{LOADING REVERBERATION AND ITS EFFECT ON THE DEFORMED MICROSTRUCTURE OF MATERIALS}

It is very difficult to determine the development of the deformed microstructure during the high strain rate loading 


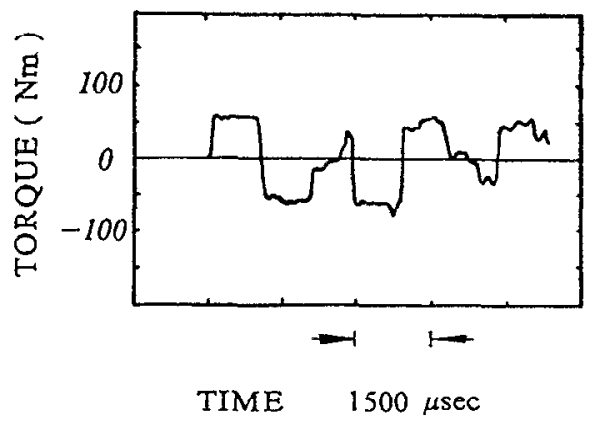

FIG. 1. A calibrating oscillogram on a standard SHTB with a dummy specimen.

process, owing to the extremely short time span. A widely used alternative method is to examine the post-mortem microstructure of the remaining plastic deformation. In such a case, a single stress pulse loading is needed. However, the standard SHTB cannot provide such a loading. The standard SHTB consists of an incident bar, a transmission bar, and a small specimen sandwiched betwcen the two long bars. Once the prestored torque in the incident bar is released to act on the specimen, the reflected and transmitted portions of the loading wave will be reflected at the two ends of the bar system. The wave reverberation back and forth along the bar system will reload the specimen repeatedly. Figure 1 shows an oscillogram with a dummy solid specimen in the standard SHTB. The wave trace recorded in the transmission bar exhibits several normal and reverse loading pulses with the same amplitude and duration as the first one. If the amplitude of the secondary loading pulse exceeds the successive yield point of the specimen, additional plastic deformation will occur and destroy the microstructure pattern of the shear localization resulting from the first stress pulse.

To examine the effect of the reverberation of torsional waves in the SHTB, a simple stress wave analysis was made, based on the following assumptions.

(1) The specimen is treated as an interface because the gauge length is much less than the length of the torsional bars.

(2) At the interface, the partition of the incident wave into reflection and transmission waves was assumed to be a constant $K=M_{t} / M_{i}$.

According to the one-dimensional stress wave theory, the torsional wave equation is

$$
\frac{\partial^{2} \theta}{\partial t^{2}}=\frac{G}{\rho} \frac{\partial^{2} \theta}{\partial x^{2}}=C^{2} \frac{\partial^{2} \theta}{\partial x^{2}},
$$

where $\theta$ is the torsional angle in unit length and $x$ is the axial coordinate along the bar. $G$ and $\rho$ are the shear modulus and the density of the bars, respectively. $C$ is the torsional wave speed. The characteristics of the waves are

$$
\tau \pm \rho c \nu=\text { const along } x \pm c t=\text { const, }
$$

where $\tau$ and $\nu$ are, respectively, the stress and the particle velocity of the sectional element adjacent to the outer surface of the bar.
In the analysis, the loading end of the incident bar is assumed to be fixed and the end of the transmission bar is assumed to be free. The physical diagram $(x, t)$ and the wave state $(\tau, \nu)$ are calculated and shown in Fig. 2. Here, $\tau_{0}$ and $\nu_{0}$ are given by

$$
\tau_{0}=\frac{1}{2} \frac{M r}{J_{b}}, \quad \nu_{0}=\frac{\tau_{0}}{\rho c},
$$

where $M$ is the prestored torque and $r$ and $J_{b}$ are the radius and the moment of inertia of the bars, respectively.

According to the wave analysis, the specimen will sustain a secondary reversal torque with the same amplitude as the first incident torque in zone 13 of Fig. 2. A comparison of the wave states between the calculation (the solid line) and the test recording (the dashed line) at point $E$ is shown in Fig. 2. The wave amplitude drop at $t^{*}$ reveals that a secondary plastic deformation must have occurred. Figure 3 is the oscillogram recorded at point $F$ and the loading reverberations of a standard SHTB test are clearly displayed.

The loading reverberation in the SHTB obscures the morphology of the deformed microstructure, which truly corresponds to the stress-strain response. But this effect has long been neglected due to the lack of an effective approach to breakup the limit of the torsional version of the Hopkinson bar, even though the post-mortem examination of the deformed microstructure has been a routine procedure in many experimental studies with this apparatus. In fact, since the secondary loading wave is a reverse pulse with the same amplitude as the first one, it will reversely deform the specimen with a similar strain rate. If the Bauschinger's effect of the material needs to be considered, the reverse secondary stress yield may be lower than the normal yield strength in the first loading. Thus, the reverse plastic deformation will readily appear. On the other hand, the rapid accumulation of heat due to the intensive local plastic deformation often results in a thermal softening effect that further reduces the yield strength of the material. In this case, the local deformed zone may retain the heat concentration to some degree after the first loading. When the secondary waves act on the specimen, the thermal softening effect may assist further plastic deformation. Hence, the loading reverberation in the SHTB becomes a significant obstacle for the quantitative investigation of the deformed microstructure, especially in the thermal-plastic shear localization case.

A typical example is the reverse kinking of the deformed microstructure in a hot-rolled low carbon steel tested on the standard SHTB for the study of shear localization behavior. In the test, the incident wave had an amplitude three times that of the transmitted one. This means that either the reflected or transmitted wave can induce secondary plastic deformation. Figure 4 gives the twisted pattern of a microstructure of shear localization due to the reloadings in the test. In a nondeformed specimen, the ferrite grains and pearlite colonies are arranged along the rolling direction and perpendicular to the shear direction. Under single wave loading, the precipitative lines of pearlites should be deformed and rotated along the shear direction. However, in Fig. 4 these lines in the center of the shear zone have been reversely distorted to their original nondeformed positions. Some of them even 


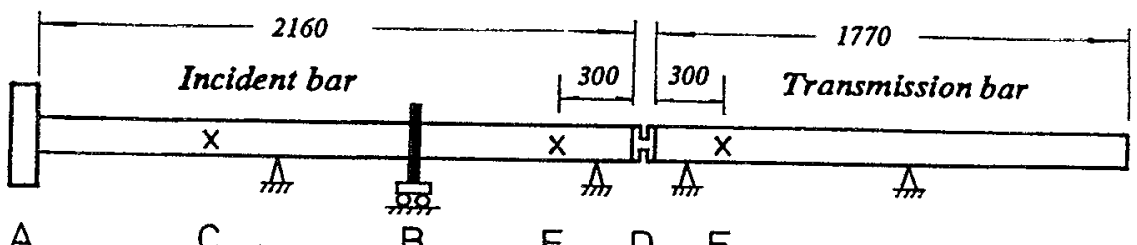

Unit $m m$

A $\quad$ C $\quad B \quad E \quad D F$

A Loading end $B$ Movable clamp C、E、 $F$ Strain rosettes $D$ Specimen

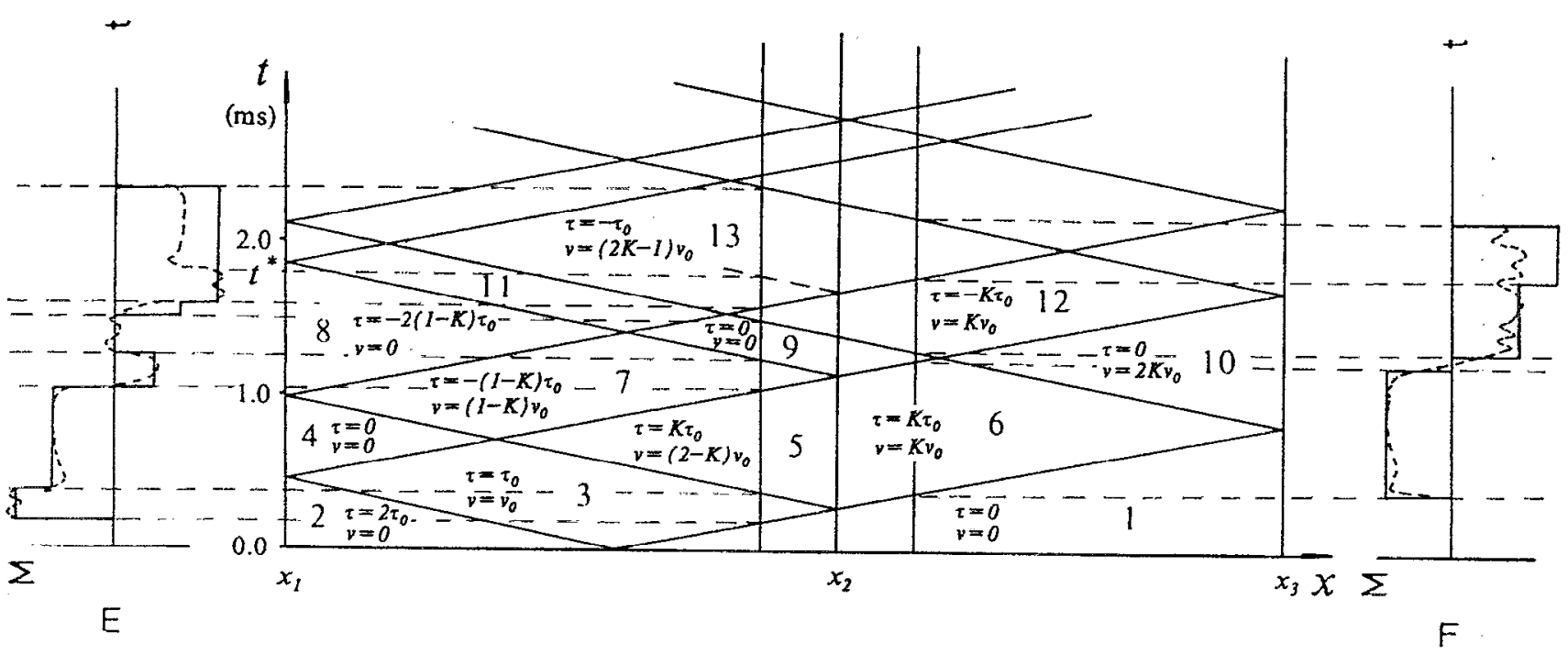

FIG. 2. A standard split Hopkinson torsional bar apparatus and its stress wave analysis.

evolve a reverse slope and form a local reverse " $\mathrm{S}$ " kinking pattern (marked by arrows in Fig. 4). Undoubtedly, the reverse loading wave should be responsible for the destroyed microstructure of shear localization. In fact, the residual image of the microstructure is the contribution made by the multiple loadings due to the loading reverberation in the SHTB. So, the loading reverberation effect in the SHTB must be eliminated to study the correlation of the macroscopic shear stress-strain curve to the microstructure of the tested material, especially for the study of shear banding.

\section{MODIFICATION OF THE SHTB}

\section{A. The principle of the modified SHTB}

The cardinal rule of the loading reverberation in the SHTB is that the residual energy cannot be released after the

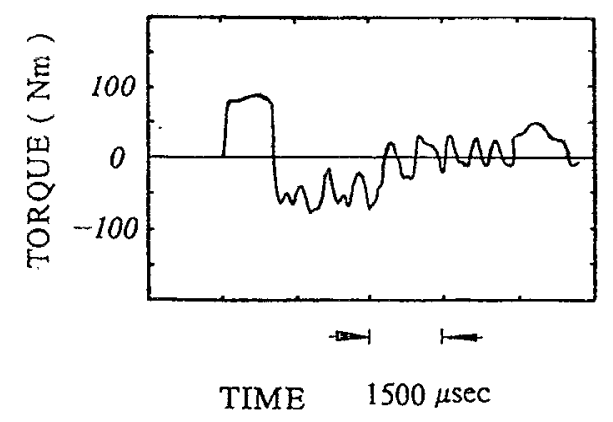

FIG. 3. An oscillogram of a test on a standard SHTB at the point F. Several subsequent loading pulses following the first one acted on the specimen. first loading. The wave analysis indicates that the secondary loading waves consist of two parts: the reflection of the reflected wave at the loading end of the incident bar, and the reflection of the transmitted wave at the free end of transmission bar. If these reflections can be excluded from the bar system, the loading reverberation effect can be completely eliminated.

Taking advantage of the concept of the flying bar (the

\section{Shear Direction}

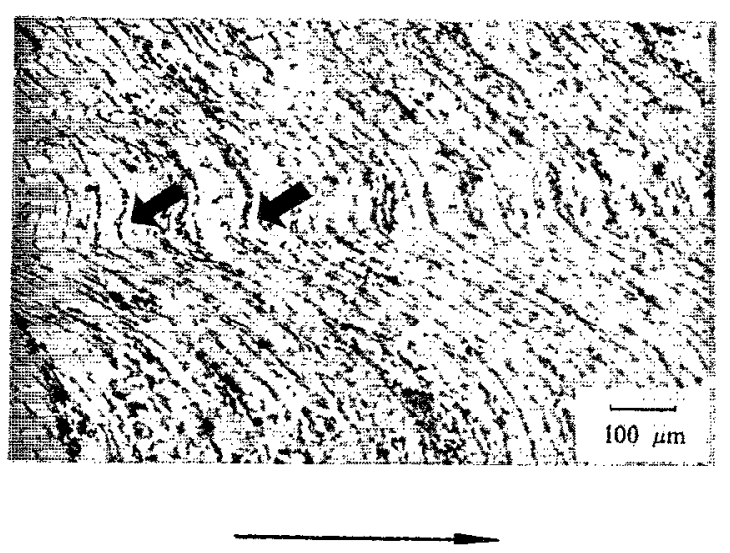

FIG. 4. A destroyed microstructure of shear localization in a hot-rolled steel due to the reverberation of loading wave of a standard SHTB (The arrows mark the reverse " $S$ " kink). 


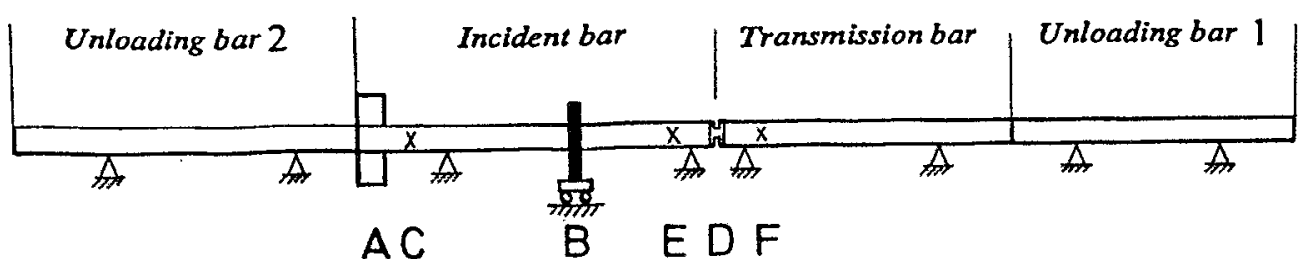

A Loading end B Movable clamp C、E、F Strain rosettes D Specimen

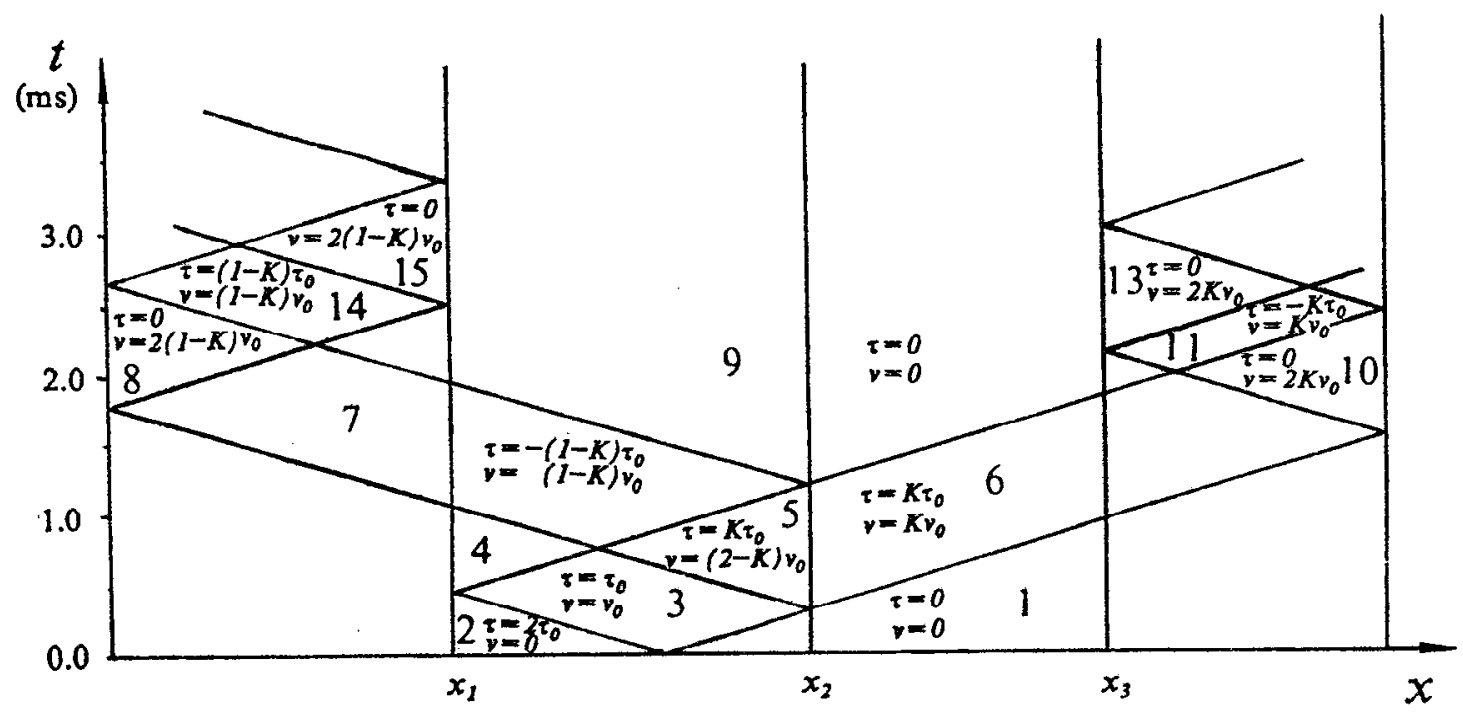

FIG. 5. A modified SHTB and its stress wave analysís.

momentum bar) in the split Hopkinson pressure bar, we suppose that two similar bars can be attached to the two ends of the main bar system. These are marked as unloading bars 1 and 2 , respectively (see Fig. 5). If the secondary waves emanating from the specimen can be completely transmitted into the two unloading bars without significant reflection effect at the interfaces, and if the unloading bars can separate from the main bar system after the secondary waves wholly enter the unloading bars--before their fronts, reflected at the rear ends of the unloading bars, return to the interfaces between the unloading bars and the main bar system-the secondary reflected waves will be isolated out of the main bar system. In order to ensure that the whole secondary waves pass into the unloading bars without returning, the length $L$ of the two unloading bars is required to be

$$
L \geqslant x_{\max },
$$

where $x_{\max }$ is the maximum distance between the clamp and the loading end in the incident bar.

By attaching unloading bars, the modified bar system becomes a four bar version. A similar stress wave analysis can be made for the new system and the wave states $(\tau, \nu)$ are shown in Fig. 5. From the analysis, it is clear that the secondary reflections are led out of the main bar system after the first loading under the above conditions.

\section{B. Procedure for the modification}

The difficulty in leading out the secondary waves rests in the connection between the unloading bars and the main bar system. It is necessary to operate with a twofold objective: to permit the unblocked transmission of the secondary waves from the main bar system to the unloading bars, and to separate the main bar system from the unloading bars at the appropriate moment.

Examining the stress wave analysis in Fig. 5, one can readily find that unloading bar 1 will rotate with an angular speed faster than the transmission bar, when the transmitted wave reflected from the free end of the unloading bar reaches the interface $x_{3}$ (see wave zone 13 in Fig. 5). In return, when the reflected wave reaches the interface $x_{1}$ from the free end of unloading bar 2, the circumstance is similar except for the reverse rotating direction (see wave zone 15 in Fig. 5). Taking this feature into account, if two connectors, transmitting the torque wave only in one direction, are used, these speed differences will allow the separation of the unloading bars from the main bar system to occur automatically.

Based on this concept, a couple of special clutches were developed to realize the connector's function. Figure 6

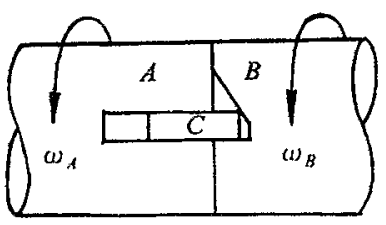

$\omega_{1} \geqslant \omega_{B}$

(a)

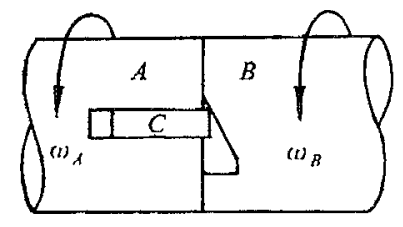

$\omega_{A}<\omega_{B}$

(b)
FIG. 6. Principle of the inside-pushed clutch. 


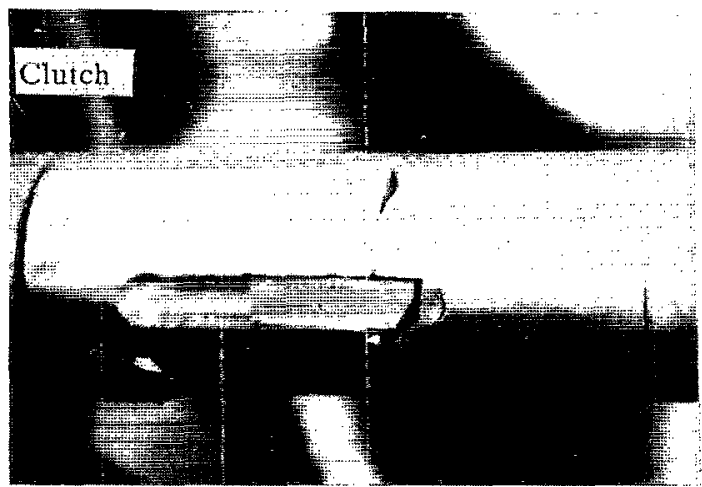

FIG. 7. Inside-pushed clutch.

shows the principle of the clutch. This clutch consists of two collars (marked as A and B) that are glued to the transmission and unloading bars, respectively. Initially the clutch is fixed as shown in Fig. 6(a). Four sliders (marked as C) put in the guide of collar $\mathrm{A}$ are inserted into collar $\mathrm{B}$. If the angular speed $\omega_{\mathrm{A}}$ is equal to or greater than $\omega_{\mathrm{B}}$, the torsional wave will propagate from collar $\mathrm{A}$ to collar $\mathrm{B}$ through the $\mathrm{C}$ sliders [see Fig. 6(a)]. Once $\omega_{A}$ becomes smaller than $\omega_{B}$, the $C$ sliders will be pushed back into the guide of collar $A$ by the slope guide of collar B [see Fig. 6(b)]. The separation between the main bar system and the unloading bars is automatically completed. Thus, the secondary waves are segregated from the bar system. This clutch, called as the insidepushed clutch from its operating principle, is shown in Fig. 7. The unloading bars associated with the special clutches are able to isolate the secondary reflected waves out of the main bar system.

\section{Redesign of the loading head}

Since the rear end of the transmission bar is free in the standard SHTB, the transmitted wave can easily be led out of the main bar system by using the unloading bar and the clutch. But the head of the incident bar in the usual design of the standard SHTB is fixed with a great inertia moment chuck for loading and calibration. The inertia moment of the chuck is so great that its effect on an oncoming wave is regarded as a fixed end. The incident bar with such a fixed head end cannot allow any reflected wave from the spccimen to pass into unloading bar 2 . On the other hand, if the head end of the incident bar were made free, the prestored torque could not be available. This dilemma seems to be the major barrier for eliminating the reloading waves. According to the stress wave analysis of the modified SHTB, the head end of the incident bar is required to keep a fixed end for the prestored torque and to become free in a reverse direction for the propagation of the reflected wave. Therefore, a special loading system was developed to transform the head end of the incident bar from fixed to free at an appropriate time. This redesigned loading system is outlined in Fig. 8. The connection between the loading head with a great inertia moment and the incident bar was fulfilled by a ratchet wheel device. The outflange with the check pawl was fixed to the loading head, whereas the inside wheel was anchored to the

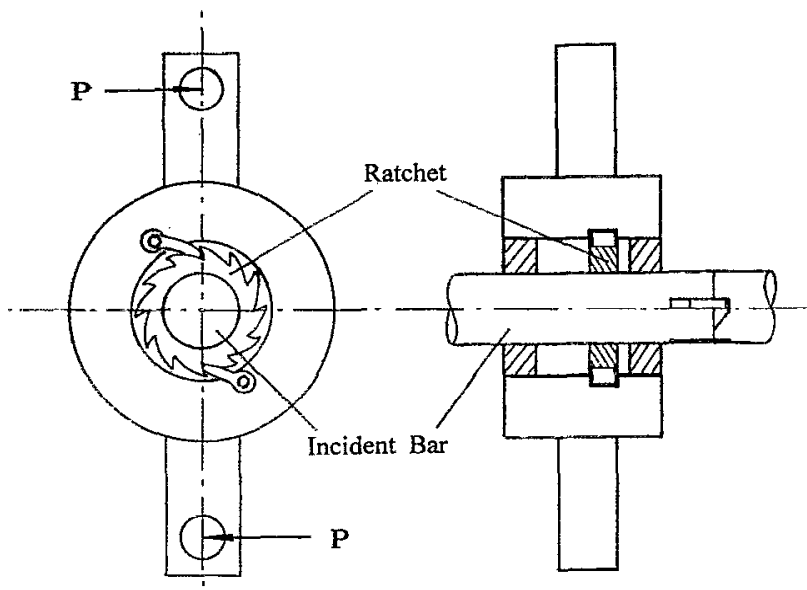

FIG. 8. Schematic view of the redesigned loading system.

incident bar. Keeping the necessary strength tolerance, the inside wheel was designed with the thickness and inertia radius kept as small as possible so as to minimize the amplitude of the induced wave reflection and its duration. The torque transmission between the inside and outside wheel was completed by the check pawl. When the loading head rotates clockwise, the check pawl drives the incident bar to store the torque in the section between the clamp and the loading head, and restrain the head end of the incident bar as a fixed end. Once the stored torque is released, the incident bar recovers its initial state and keeps its rotating speed at zero. Until the reflected wave from the specimen reaches $x_{1}$, and makes the loading end of the incident bar rotate in the reverse direction, the reflected wave can propagate into unloading bar 2 . Finally, unloading bar 2 and the clutch arrest the reentry of the secondary reflected wave in unloading bar 2 into the main bar system. Thus, the loading reverberation in the SHTB can be completely eliminated.

\section{RESULTS AND DISCUSSION}

For the current standard SHTB in our laboratory, the maximum distance of the stored torque section of the incident bar (between the clamp and the loading head) is 1660 $\mathrm{mm}$. According to the above analysis, the two unloading bars were chosen to be 2160 and $1780 \mathrm{~mm}$ in length, respectively, longer than the stored torque section. The unloading bars with $25 \mathrm{~mm}$ outside diameter were made of a Ly 12 aluminum alloy (similar to $2024 \mathrm{Al}$ ) as were the main bars. Figure 9 gives the oscillogram of dynamic calibration on the modified SHTB with a dummy specimen. In the oscillogram, as anticipated, only one loading pulse is observed except for several disturbances about one-third of the amplitude of the transmitted wave. They may be attributed to the wave reflection arising from the clutch's unperfect contact.

In order to determine the effectiveness of the modification, a contrast examination was carried out with the modified SHTB under the same test conditions, which resulted in the destroyed microstructure in Fig. 4. The wave amplitude, the tested material, and the dimensions of the specimen were similar to the previous examination. Figure 10 gives the oscillogram recorded at $F$ in the modified SHTB. It is clear that 


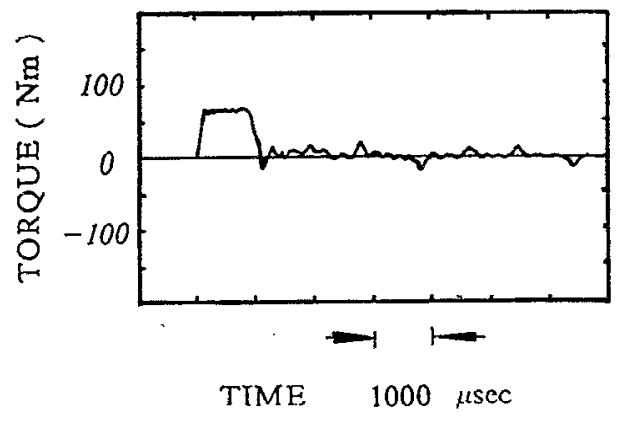

FIG. 9. A calibrating oscillogram on the modified SHTB with a dummy solid specimen.

the subsequent small loading pulses have about one-third or one-half the amplitude of the transmitted pulse, although the reflected wave possesses an amplitude two times higher than the transmitted wave. The residual small waves may result from the reflection of interfaces between the sliders and the guiders. Figure 11 shows the corresponding microstructure of shear localization in the contrast test. The precipitation of pearlite distinctly displays the shear localization zone, in which the maximum shear strain in the center of the shear band reaches 3.5. The microstructure of the mature shear band deformation shows no trace of reverse plastic deformation and kinking pattern. This result indicates that the modification of the standard SHTB does eliminate the loading reverberation effect on the post-mortem observation of the deformed microstructure in the tested material. So, the modified SHTB can ensure the ability to study the correlation of the transient stress-strain curve to the microstructures and microdamages of the deformation process.

The loading reverberation phenomenon exists in many dynamic loading apparatus. For example, in the direct tensile version of the split Hopkinson bar and in the plate impact test on a light-gas gun. In fact, any wave loading system with a fixed small specimen sandwiched between two long bars has such a problem. Noticing the damage caused by reloading, Eleich and Campbell (1976) ${ }^{11}$ used a reverse torsion shear technique in the SHTB to examine the reverse reloading effect on a $\mathrm{Mg}-\mathrm{Al}$ alloy and pointed out that the weakening effect of the yield stress increased with the reloading strain rate. Early investigators focused their attention on the dynamic mechanical response of materials. The microscopic observation of tested materials only played a supplementary role. In the last decade, with the development of tracing the inherent mechanism of large ductile deformation under high

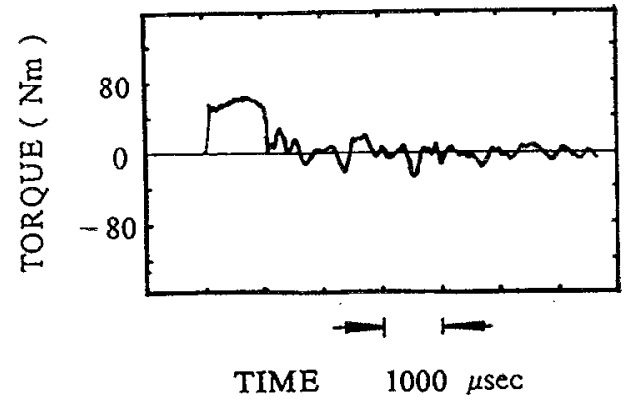

FIG. 10. An oscillogram of a test on the modified SHTB at the point F.
Shear Direction

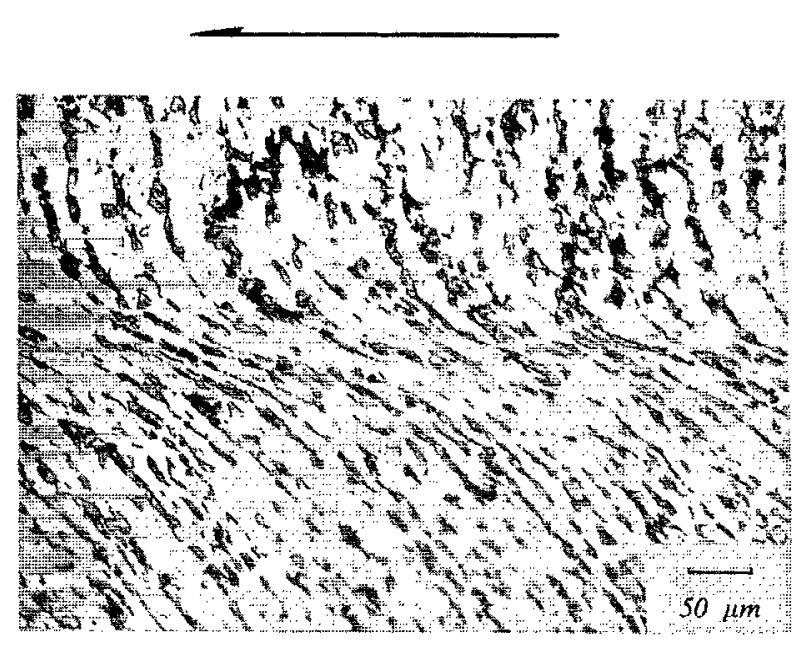

FIG. 11. A perfect pattern of the shear band microstructure obtained in a test on the modified SHTB under the same conditions as the destroyed one.

rates, the studies involving microstructures and microdamage became more and more important. However, because of the lack of effective approaches to remove the reloading effects, one had to neglect this effect first when examining the postmortem microstructure. In order to relate the microstructural evolution to the loading history, a novel modification of the Hopkinson tension bar was made by Nemat-Nassar ${ }^{12}$ for a recovery experiment. A momentum trap bar was used to isolate the reflected wave in a direct tension experiment. As for the torsional version of the Hopkinson bar, it is more difficult to solve the problem due to its complex structure and the type of wave propagation. Xue et al. $(1992)^{10}$ discussed this harmful effect of loading reverberation on the post-mortem observation for the deformed microstructure, when they investigated the evolution of the microstructure during shear banding process. An improvement had been made to remove the reflection of the transmitted wave. However, that work cannot eliminate the reloading of the reflected wave. Thus, the amplitude of the loading wave had to be limited to keep the stress induced by the reloading of the reflected wave lower than the secondary yield stress of the material. This condition restrained the loading strain rate to a certain extent. In the current study, based on the above-mentioned improvement, the SHTB technique was modified further, including the redesign of its loading system and the attachment of two unloading bars and two special clutches. The new SHTB can lead the reflected wave and the transmitted wave out of the main bar system and thus can realize the single wave pulse loading on the specimen. This guarantees the availability of a macromicroscopic correlating study without any subsidiary limitation.

\section{ACKNOWLEDGMENT}

This work was supported by the Chinese National Science Foundation. 
${ }^{1}$ W. E. Baker and C. H. Yew, J. Appl. Mech. Trans. ASME(E) 33, 917 (1966).

${ }^{2}$ H. Kolsky, Proc. Phys. Soc. B 62, 676 (1949).

${ }^{3}$ J. D. Campbell and A. R. Dowling, J. Mech. Phys. Solids 18, 43 (1970).

${ }^{4}$ T. Nicholas, Exp. Mech. 11, 370 (1971).

${ }^{5}$ J. Duffy, J. D. Campbell, and R. H. Hawley, J. Appl. Mech. Trans. ASME(E) 38, 83 (1971).

${ }^{6}$ R. A. Frantz and J. Duffy, J. Appl. Mech. Trans. ASME(E), 39, 939 (1972).

${ }^{7}$ J. Lipkin, J. D. Campbell, and J. D. Swearengen, J. Mech. Phys. Solids 26, 251 (1978).
${ }^{8}$ A. Gilat and Y. H. Pao. Exp. Mech. 28, 322 (1988).

${ }^{9}$ L. Anand, O. Dillon, T. A. Place, and B. F. Von Turkovich, Report of the NSF Workshop on Localized Plastic Instabilities and Failure Criteria [Int. J. Plast. 6, I (1990)].

${ }^{10} \mathrm{Q}$. Xue, Y. Bai, and L. Shen, in Proceedings of the Second International Symposium on Intense Dynamic Loading and Its Effects, edited by Zhang Guanren et al. (Sichuan University Press, Chengdu, China, 1992), p. 405.

${ }^{11}$ A. M. Eleiche and J. D. Campbeil, Exp. Mech. 16, 281 (1976).

${ }^{12}$ S. Nemat-Nassar; in Shock-Wave and High-Strain Rate Phenomena in Materials, edited by M. A. Meyers et al. (Marcel Dekker, 1992), p. 1. 